\title{
POLÍTICAS DE INNOVACIÓN SOCIAL Y DISEÑO: ENTRETEJIDO QUE CONSTRUYE LA PROYECCIÓN SOCIAL DE LA ACADEMIA.
}

\author{
SOCIAL POLICY INNOVATION AND DESIGN WEAVING \\ THAT BUILDS ACADEMY'S SOCIAL PROJECTION
}

\section{JOHANNA ZÁRATE HERNÁNDEZ}

Diseñadora Gráfica, Especialista en Edición Digital y Multimedia, aspirante al título de Maestra en Estética e Historia del Arte. Profesor asociado 1, tiempo completo, Universidad Jorge Tadeo Lozano (Bogotá, Colombia). johanna.zarate@utadeo.edu.co.

\section{JUAN MANUEL ESPAÑA ESPINOZA}

Diseñador Industrial, Especialista en Gerencia de diseño, magister en Gestión Ambiental. Profesor asociado 2, Tiempo completo, Universidad Jorge Tadeo Lozano (Bogotá, Colombia).

\section{SUSANA BALLESTEROS OYAGA}

Estudiante Universidad Jorge Tadeo Lozano, Programa de Diseño Industrial (Bogotá, Colombia).

\section{RESUMEN:}

Durante la primera década del siglo XXI el concepto de innovación social ha aumentado su auge en los estamentos académicos, empresariales, gubernamentales y no gubernamentales, aparecen múltiples estudios, análisis, proyectos e iniciativas de todo tipo que abordan este tema, lo definen en busca de soluciones viables para las diferentes problemáticas que aquejan a múltiples entornos en los cuales se desarrollan los seres humanos en la actualidad. Dentro de este nuevo y creciente interés, la Universidad Jorge Tadeo Lozano y el programa de Diseño Industrial dedican tiempo y recursos significativos en lo que denominan "proyección social". Dentro del contexto se propuso un proyecto que permite aprovechar una red construida de forma aleatoria y empírica para estructurar la construcción de una red y comunidad académica enfocada en la proyección social y la innovación en diseño en la Universidad Jorge Tadeo Lozano, siendo esta una reflexión sobre las políticas que aquí aplicaron.

\section{PALABRAS CLAVE:}

Innovación social, comunidad académica, red.

\section{ABSTRACT:}

During the first decade of the century the concept of social innovation has increased its boom in academic, business, government and non- government bodies, appear multiple studies, analyzes, projects and initiatives of all kinds that address this issue, define it in search of workable solutions to the various problems afflicting multiple environments in which human beings develop today. Within this new and growing interest, the University Jorge Tadeo Lozano and Industrial Design program devoted significant time and resources in what they call "social projection". Within the context of a project that takes advantage of the network randomly constructed empirically to structure and build a network and community focused on academic outreach and innovation in design at the University Jorge Tadeo Lozano, this being a reflection on proposed policies applied here.

\section{KEYWORDS:}

Social innovation, academic community, network. 
ARTE \& DISEÑO, ISSN: 1692-8555, Vol. 11 N² 2, Julio - Diciembre 2013 JOHANNA ZÁRATE HERNÁNDEZ

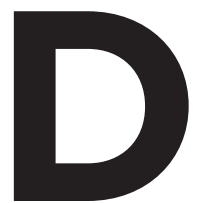

urante la primera década del siglo XXI el concepto de innovación social ha aumentado su auge en los estamentos académicos, empresariales, gubernamentales y no gubernamentales; tan es así, que aparecen múltiples estudios, análisis, proyectos e iniciativas de todo tipo que abordan este tema, lo definen y redefinen en busca de soluciones viables para las diferentes problemáticas que aquejan a los múltiples entornos en los cuales se desarrollan los seres humanos en la actualidad.

Dentro de este nuevo y creciente interés por la innovación social, la Universidad Jorge Tadeo Lozano y el programa de Diseño Industrial dedican tiempo y recursos significativos en lo que denominan "proyección social". Dentro del contexto de esta aproximación desde la academia a la proyección social se hace evidente la aparición casi espontánea de una red de contactos, proyectos, ideas y colaboraciones que nacen desde diferentes instancias y momentos dentro del proyecto educativo institucional. Es así que se propuso un proyecto que permita aprovechar dicha red construida de forma aleatoria y empírica para estructurar la construcción de una red y comunidad académica enfocada en la proyección social y la innovación en diseño en la Universidad Jorge Tadeo Lozano. Aquí nos proponemos postular una conexión de las diferentes visiones encontradas sobre el concepto de innovación social enunciados por el Gobierno nacional, la Universidad y el programa de D.I., que sirven para dar una visión más clara sobre lo que se propone esta red, cuáles son sus límites y sus alcances y cómo busca articular diferentes entes que estén en sincronía sobre este tema.

\section{DESARROLLO}

Parece pertinente iniciar de lo macro a lo micro; se esboza inicialmente lo que postula el Gobierno de Colombia en su texto “Bases conceptuales de una política de innovación social", publicado en agosto de 2013 en la ciudad de Bogotá y realizado por el Departamento Nacional de Planeación, el Departamento Administrativo de Ciencia, Tecnología e Innovación - Colciencias y la Agencia Nacional (DNP), para la Superación de la Pobreza Extrema (ANSPE). Este documento explica que dentro del Plan Nacional de desarrollo 2010 - 2014 “Prosperidad para todos" será la innovación la pieza central y clave para actividades productivas, procesos sociales de colaboración público privada, gestión del desarrollo sostenible, progreso social, buen gobierno y consolidación de la paz en Colombia. Para lograr cumplir con este plan, el gobierno de Colombia incorpora el concepto de innovación social en el marco de la política nacional de ciencia, tecnología e innovación, en busca de lograr una sociedad equitativa, incluyente, próspera y en paz. Cabe resaltar la actual postura estatal que además de permitir, promueve el trabajo colaborativo en pro de cumplir con las metas comunes que se plantean en los planes de innovación social (IS):
"De otro lado, el surgimiento de un sector público no estatal (ONG's) cada vez más fuerte, motivado por la reducción del estado de bienestar y las dificultades de cobertura, el surgimiento del "Estado relacional" y la evolución de la Responsabilidad Social Empresarial, que ha conllevado a la generación de empresas y emprendimientos sociales, han configurado un nuevo panorama donde la IS es necesaria para la solución creativa de los problemas sociales, económicos y ambientales, y pone de relieve la importancia del trabajo colaborativo entre diferentes agentes de la sociedad, especialmente aquellos que se ven directamente afectados por ellos."(DNP, Colciencias, ANSPE, 2013).

Es importante en este análisis dejar en claro que al hablar de innovación e incluir lo social, interesa destacar el hecho de que este proceso se da al margen de los procesos económicos o empresariales productivos tradicionales; no por eso se desconoce la importancia de los mismos, solo que se busca una aproximación diferente a problemas que han excedido la capacidad productiva, económica o política tradicional de ser solucionados. Con respecto a la visión o el enfoque que la política pública le da al concepto de innovación, se afirma:

Por innovación, en sentido amplio, se entiende la capacidad y el proceso de generación, transferencia y aplicación de conocimiento científico y/o empírico, dentro y fuera del ámbito del mercado, que incremente la productividad o la efectividad de una comunidad, organización o empresa en forma sostenible, permitiéndole alcanzar sus objetivos, satisfacer sus necesidades o incrementar su bienestar. Es decir, la innovación crea valor a partir del conocimiento y se generan externalidades positivas de carácter económico y social. (DNP, Colciencias, ANSPE, 2013).

Esta visión estatal de la innovación nos interesa, ya que contempla, en primer lugar, el concepto de lo colaborativo al tener en cuenta los vínculos y lazos que se construyen con ella; son estos aspectos los que la hacen social per se. Si se tienen claras las dinámicas que la IS conlleva, es clara entonces su relación con el conocimiento, ya sea científico o empírico; y es esto muy relevante cuando hablamos de participación, colaboración e igualdad, aspectos fundamentales en la consolidación de una red y una comunidad de cualquier tipo. Ahora a este enfoque del concepto de innovación se le debe también sumar el de lo social, que en este caso postula la misma política pública después de analizar, comparar y sopesar diferentes enfoques, como el sistémico, sociológico, el pragmático, el económico, el gerencial, el enfoque de la ciencia política y el participativo. 


\begin{abstract}
"Si entendemos lo "social" como el espacio generado por y para la convivencia de personas y grupos, nos referimos, fundamentalmente, a problemas $y$ oportunidades de relaciones que son económicas, sociales, políticas o culturales. Cuando tales relaciones no funcionan adecuadamente hay espacio para pensar en nuevas ideas, básicamente involucrando a cuatro actores clave: la comunidad, el Estado, el sector privado y el sector no estatal sin ánimo de lucro. Por lo tanto, es distintivo de la IS que parta de iniciativas que emergen desde las organizaciones de la sociedad civil, del sector social y la política pública entre otras". (DNP, Colciencias, ANSPE, 2013).
\end{abstract}

Al referirse al sector no estatal sin ánimo de lucro es posible entonces incluir a la academia, que aunque no aparece en forma explícita, debe ser entendida en forma tácita, ya que la academia puede ser, una catalizadora de los saberes y el conocimiento científico y empírico, promover y generar nuevas ideas y vincular a los actores arriba mencionados. Si se tiene en mente que estos actores deben tener objetivos en común y suponemos que todos buscan solucionar necesidades o problemas sociales como la educación, el empleo, la salud, la justicia y la democracia, el cuidado del medio ambiente y el hambre, podemos recalcar que "... el objeto de la IS se localiza dentro y fuera del mercado" (DNP, Colciencias, ANSPE, 2013), ya sea en entornos regionales o locales. Estos entornos en los cuales se desarrollan los proyectos de IS deberían siempre tener en cuenta el trabajo con innovación en la comunidad, en el cual se tenga presente el conocimiento empírico de los actores de la comunidad, quienes tienen claridad sobre necesidades propias, organización de cada grupo social, estructura, y según sea el caso, capacidad de producción técnica de la comunidad. De esta forma, las características ideales de la IS son la novedad, el potencial de éxito, la sostenibilidad y la participación de la comunidad directamente involucrada. De otro lado, es altamente deseable para la política pública que estos proyectos de IS posean potencialidad para la política pública, permitan el intercambio y la transferencia contextualizada de conocimiento y experiencia y que sean económicamente favorables para todos los actores involucrados. En resumen, para la política pública de Colombia la IS se define como

"Es el proceso a través del cual se crea valor para la sociedad mediante prácticas, modelos de gestión, productos o servicios novedosos que satisfacen una necesidad, aprovechan una oportunidad y resuelven un problema social de forma más eficiente y eficaz que las soluciones existentes, produciendo un cambio favorable en el sistema en el cual opera.

La innovación social se caracteriza por tener potencial de escalabilidad, replicabilidad, ser sostenible, promover mayores niveles de empoderamiento de la comunidad y generar alianzas entre diferentes actores de la sociedad". (DNP, Colciencias, ANSPE, 2013).

Teniendo ya algo de claridad sobre la postura nacional sobre la IS, podemos pasar a revisar las posturas institucionales con respecto a la investigación, creatividad e innovación, la proyección social y la internacionalización, que aunque no se enfoca directamente en la proyección social, es un aspecto con el cual los proyectos de IS de la Universidad y la red en específico pueden contribuir. Sobre la proyección social el Proyecto Educativo Institucional (PEI) propone, entre otras:

1. Privilegiar la responsabilidad social como parte fundamental de los objetivos académicos en su relación con el medio externo, con el propósito de contribuir al desarrollo de la sociedad y del país.

2. Promover la participación institucional en organizaciones que permitan desarrollar proyectos y actividades de impacto social que contribuyan al mejoramiento de la calidad de vida de la población, así como en la construcción y consolidación de vínculos con diferentes actores e instituciones sociales.

3. Promover prácticas sociales, culturales, investigativas, empresariales y de procesos creativos, así como consultorías y asesorías que fortalezcan la relación de la universidad con el entorno y con otros actores e instituciones de la vida nacional.

4. Promover espacios de investigación, formación, participación, diálogo y reflexión entre la comunidad tadeísta y comunidades externas para la construcción conjunta de soluciones creativas, novedosas y viables a problemáticas sociopolíticas, económicas y medioambientales de nuestra sociedad.

Aquí se empiezan a encontrar puntos comunes entre las políticas nacionales y las institucionales que sirvan de base fundacional para una red más estructurada. Estos puntos pue-den enunciarse como:

Ahora, teniendo ya esta comparación, podemos dar paso a la revisión de las políticas del Proyecto Educativo del Programa Académico (PEPA), de Diseño Industrial de la Universidad Jorge Tadeo Lozano para identificar cómo se relacionan con las anteriores, y así definir las políticas internas ideales de una red y comunidad académica enfocada en la proyección social y la innovación en diseño en el contexto institucional. Dentro del perfil del diseñador industrial tadeísta se plantea que dentro del programa

... se diseñan espacios formativos que sobrepasan las posibilidades del aula y proponen a la comunidad educativa hacia escenarios alternativos que convocan 


\section{POLITICA NACIONAL}

Solución creativa de los problemas sociales, económicos y ambientales.

Generación, transferencia y aplicación de conocimiento científico y/ o empírico de la sociedad

\section{POLITICA INSTITUCIONAL}

Construcción conjunta de soluciones creativas, novedosas y viables a problemáticas socio-políticas, económicas y medioambientales de nuestra sociedad.

Privilegiar la responsabilidad social como parte Trabajo colaborativo entre diferentes agentes

fundamental de los objetivos académicos en su relación con el medio externo, con el propósito de contribuir al desarrollo de la sociedad y del país.

Promover espacios de investigación, formación, participación, diálogo y reflexión entre comunidad Tadeísta y comunidades externas

Consultorías y asesorías que fortalezcan la relación de la Universidad con el entorno

Incremento de la productividad o la efectividad de una comunidad, organización o empresa en forma sostenible

Prácticas, modelos de gestión, productos o servicios novedosos que satisfacen una necesidad, aprovechan una oportunidad y resuelven un problema social de forma más eficiente y eficaz que las soluciones existentes
Promover la participación institucional en organizaciones que permitan desarrollar proyectos y actividades de impacto social que contribuyan al mejoramiento de la calidad de vida de la población, así como en la construcción y consolidación de vínculos con diferentes actores e instituciones sociales.

Fuente: elaboración propia de Zárate (2013).

Cuadro 1: Nodos comunes entre políticas macro que soportan la red.

reflexiones críticas, creativas y propositivas sobre la producción de diseño industrial... estas reflexiones se correlacionan con escenarios sociales significativos para el Diseño Industrial en la actualidad: espacios públicos; espacios interactivos de información, instituciones culturales; espacios de producción industrial, manufacturera o artesanal; espacios empresariales; espacios habitados por comunidades locales quienes promueven proyectos de emprendimiento o por comunidades en situación de vulnerabilidad. (PEPA, 2013).

Dentro del perfil del Diseñador Industrial Tadeísta y la particularidad de su proyecto académico que define tres rutas de enfoque denominadas 0bjeto, Interacción y Contexto, se desarrolla la Plataforma de Investigación, creatividad e innovación a la cual la red se propone apoyar y visibilizar, teniendo claridad que dentro del perfil profesional del egresado “... se asume como obligatorio, en el marco del Proyecto Académico del Programa de Diseño Industrial, reflexionar sobre la pertinencia profesional y acción social del Diseño Industrial" (PEPA, 2013) se hace necesario apoyar el desarrollo de dicho perfil, mediante el desarrollo de proyectos dentro del grupo de investigación del programa, que promueve la generación de espacios de reflexión sobre la pertinencia, relevancia y trascendencia de la innovación social, que permitan el desarrollo de procesos de innovación y creación desde el diseño. Dentro de los lineamientos del programa en proyección social podemos resaltar aquellos que interesan aquí directamente:

1. El programa busca proponer, promover y apoyar el trabajo de impacto social positivo a través de programas y proyectos que vinculen a los diferentes miembros de la comunidad académica del Programa, y que permitan la obtención de resultados y productos acordes con procesos 


\section{MOMENTO}

Construcción proceso
y grupo estructural de
desarrollo

OBJETIVO

Creación plan de trabajo, socialización del proyecto en todos los sectores y construcción de alianzas estratégicas.

Construir la realidad del colectivo y
Marco de realidad

Marco funcional Diseñar el sueño colectivo a
Plan marco estratégico
desarrollar en el futuro en el territorio.
A puestas, objetivos, alcances y
logros. del territorio actual a partir de las variables fundamentales.

Construir la funcionalidad pensada, tanto del colectivo como del territorio, a partir de las variables fundamentales.

Construir la base del sueño colectivo, 5 talleres, 30 Marco estratégico a desarrollar a partir de las propuestas estratégicas. personas cada uno. Total 150 personas.

\section{PRODUCTO}

Diseño y gestión de
plan de trabajo

Plan de Gestión del Proyecto Visión Cartagena

20 talleres, 50

personas cada uno. Documento Marco Total 1000 de Realidad. personas.

20 talleres, 50 personas cada uno. Documento Marco Total 1000 Funcional. personas.

5 talleres, 30 personas cada uno. Total 150 personas.

\section{Documento Marco Estratégico.}

Marco Estratégico.
Documento Plan

\section{MOMENTO}

Construcción proceso

y grupo estructural de desarrollo

\section{OBJETIVO}

Creación plan de trabajo, socialización del proyecto en todos los sectores y construcción de alianzas estratégicas.

\section{ACCIÓN}

Diseño y gestión de plan de trabajo
Plan de Gestión del

Proyecto Visión

Cartagena de alta calidad y a los requerimientos de Colciencias. Entendiendo como positivo todo trabajo que busque contribuir al desarrollo del país fortaleciendo las capacidades de los sectores productivos y grupos humanos, y aportar en el mejoramiento de su calidad de vida.

2. En armonía con el Proyecto Educativo Institucional, el componente de proyección social del programa fortalece la integración de profesores, estudiantes y egresados, mediante actividades y proyectos disciplinares e interdisciplinares, intra e interinstitucionales.
3. Acorde con los principios de la Universidad Jorge Tadeo Lozano, que buscan el fortalecimiento de los lazos entre la comunidad académica y la sociedad en pro de su desa-

4. rrollo, el programa promueve y fortalece espacios formativos que vinculen a organizaciones tanto del sector público como privado mediante acuerdos, convenios y alianzas.

5. El componente promueve y gestiona la articulación de actores, recursos y espacios de los proyectos de proyección social aprobados por el comité académico del programa con el fin de potenciar su impacto positivo en los entornos so- 
ARTE \& DISEÑO, ISSN: 1692-8555, Vol. 11 N² 2, Julio - Diciembre 2013

JOHANNA ZÁRATE HERNÁNDEZ

ciales, culturales, comerciales, ambientales y empresariales.

6. Los proyectos se enmarcan en cinco programas: Talleres de investigación, creación, innovación (con organizaciones), prácticas universitarias, consultorías, talleres verticales, apoyo a investigación, creación e innovación.

7. Se requiere que las instituciones y actores externos a la universidad formalicen su vinculación al proyecto mediante convenios marco y específicos, y en la medida de lo acordado movilicen recursos para la ejecución; los proyectos deben estar cofinanciados.

8. Se busca fomentar la continuidad en el tiempo de los proyectos, y su conexión con los espacios de investigación, como una forma de aumentar su impacto tanto académico como social.

Si se tienen estos como los principales lineamientos de la proyección social dentro del programa y que hasta el momento han sido la guía de los proyectos en curso, nos permitimos complementar el cuadro que une los nodos comunes de las diferentes políticas macro y micro que interesan aquí. Esta revisión permite, pues, generar un marco de referencia global en cual se enmarque la red de forma práctica, para que sea un ente viable y que apoye y fortalezca los proyectos, propuestas y políticas ya existentes.

Resultado de comparar estas políticas y lineamientos son el sustento y los lineamientos que soportan el resultado de este proyecto, que es la puesta en marcha de la red y comunidad académica enfocada en la proyección social y la innovación en diseño en la Universidad Jorge Tadeo Lozano. Esta red, teniendo en cuenta los principios de formación del Programa, permitirá "Fomentar la conciencia entre los diseñadores industriales sobre la responsabilidad de la profesión y su ejercicio -deontología- en el marco de las dinámicas de intercambio social, económico, político, cultural y ecológico de la actualidad" (PEPA, 2013). Es así como la red permitirá cumplir, en cierta medida, con el objetivo de "impulsar, a través de la Plataforma de Investigación, Creatividad e Innovación y sus diferentes modalidades, espacios de interacción con el medio para que los estudiantes de todos los niveles de formación del programa desarrollen sus perfiles particulares (PEPA, 2013), en este caso el perfil de aquellos interesados en la IS. Cuando se revisa la fundamentación de la proyección social en el Programa se evidencian la relación que tendrían la proyección social, la gestión y la red como herramientas que visibilizan los proyectos en curso.

\footnotetext{
"La proyección social responde al compromiso ético y social de la institución como espacio de formación que debe favorecer la conciencia crítica de la sociedad y los procesos de construcción de la realidad humana y sus dimensiones cultural, política, económica y deon-tológica (ética referida al ejercicio profesional) "
}

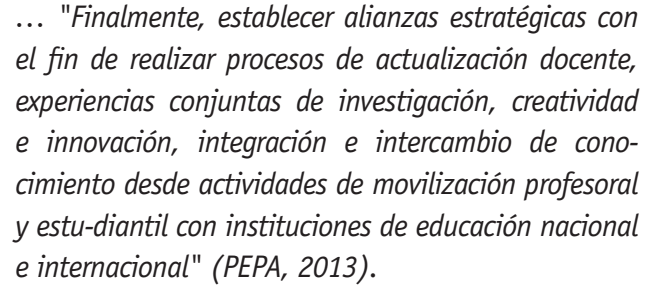

Para materializar todo lo planteado en las políticas anteriores y poder estructurar las actividades, proyectos y planes que propone el Programa de Diseño Industrial, la red puede apoyar, divulgar, proponer y generar actividades enfocadas en la IS, tales como:

- Workshops

- Talleres

- Prácticas sociales voluntarias

- Vinculaciones con proyectos de grado

- Proyectos de investigación, creatividad e innovación

- Seminarios (presenciales o en línea) con invitados internacionales

- Foros y conferencias presenciales o en línea

- Espacios académicos en segunda lengua

- Concursos internacionales de diseño enfocados en la IS

Estas actividades, que son sustentadas sobre la Plataforma de Investigación, Creatividad e Innovación, cuyo objetivo es “la organización de las acciones propuestas con los espacios curriculares y extracurriculares del programa; esta plataforma promueve y gestiona actores, recursos y espacios de los proyectos de proyección social, aprobados por el programa a fin de potenciar el impacto positivo en los entornos sociales, culturales, comerciales, ambientales y empresariales" (PEPA, 2013), serán algunas de las herramientas por desarrollar. Los aspectos descriptivos y las directrices de procedimiento de cada una de las actividades se encuentran detalladas en el documento "Lineamientos y mecanismos de proyección social en el programa de Diseño Industrial", realizado por los docentes del componente de proyección social del Programa. De esta manera, teniendo claro el perfil ocupacional del diseñador industrial tadeísta se tendrían como ejes temáticos esenciales para la red y la comunidad académica enfocada en la proyección social, los siguientes temas:

- Gestión cultural

- Salud

- Educación

- Trabajo

- Ética y responsabilidad social

- Hábitat y vivienda

- Movilidad

- Productividad industrial, manufacturera y artesanal 
Tanto los temas como las actividades antes enunciadas y que aparecen en el PEPA del Programa apoyan no solo a las políticas de IS y proyección social del Estado, la universidad y el programa, sino que también se relacionan directamente con los planes de internacionalización de la universidad y del programa, cuyo propósito es:

" Conectar a la comunidad académica del Programa de Diseño Industrial (Docentes, Estudiantes y Administrativos) con las nuevas dinámicas de orden internacional, que funcionan bajo una diversidad de discursos, los cuales ya no están limitados por fronteras geográficas o idiomáticas. Esto con el fin de que la comunidad académica tengan la oportunidad de incorporar a su formación profesional y personal algunos aspectos de vida desde perspectivas globales y experiencias multiculturales. (Componente de internacionalización, 2014)".

Así, esta red y comunidad académica tienen como resultado esperado permitir el intercambio y transferencia de conocimientos que sean productos de resultados parciales o finales de investigaciones aprobadas, artículos de divulgación como comunicación del conocimiento, resúmenes divulgativos de eventos científicos, capítulos de tesis de doctorado, maestría y trabajos de grado de pregrado, artículos de difusión sobre actividades de semilleros, artículos de divulgación de proyectos, todos previamente publicados, y programas de investigación con participación ciudadana, plataformas de proyección social y experiencias académicas enfocadas en la proyección social y actividades de innovación, que puedan utilizar esta red como un mecanismo de apoyo, divulgación e intercambio de información mediante una estructura comunicativa horizontal, que propicie la interacción de investigadores, nuevos investigadores, investigadores en formación, docentes, estudiantes, administrativos y empresarios, interesados en construir conocimiento mediante un diálogo que viabilice la búsqueda o la creación de posibles respuestas o soluciones a temáticas o problemáticas comunes.

\section{CONCLUSIONES}

Queda claro, entonces, que la red y la comunidad académica no pretenden ser una publicación en línea, sino un lugar común en el cual realizar encuentros en busca de fines comunes que propendan por la materialización de las políticas de IS que necesitan el programa, la universidad y el país.

Para cumplir con tan ambicioso plan se pretenden estructurar red y comunidad de la siguiente manera:

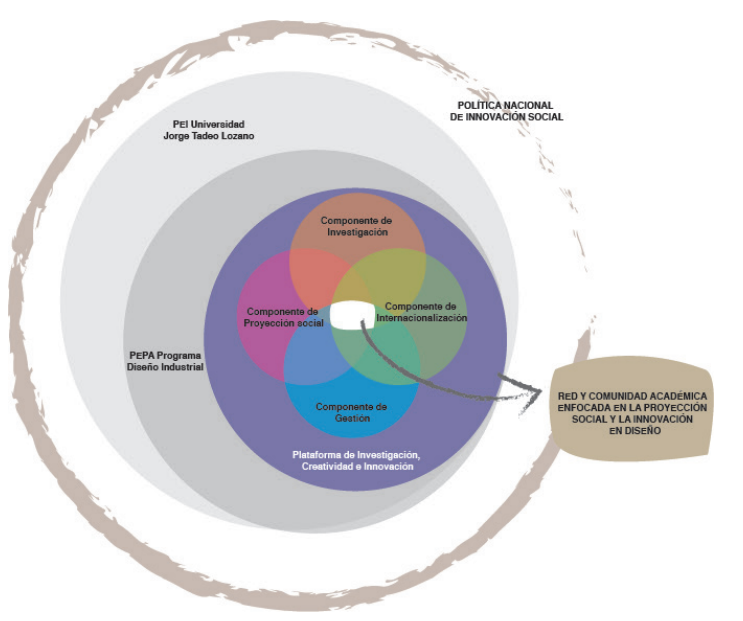

Fuente: Elaboración propia de Zárate (2013).

Imagen 1: Estructura relacional de la Red con las políticas y componentes involucrados. mación.

Con este gráfico se busca clarificar cómo esta red y comunidad académica pretende ser una herramienta integradora de los componentes que caracterizan al programa en cumplimiento con el PEI de la universidad. Ahora tendríamos que detallar un poco más las relaciones internas que se generan entre los componentes que engranan en la red y el resto de la estructura del Programa.

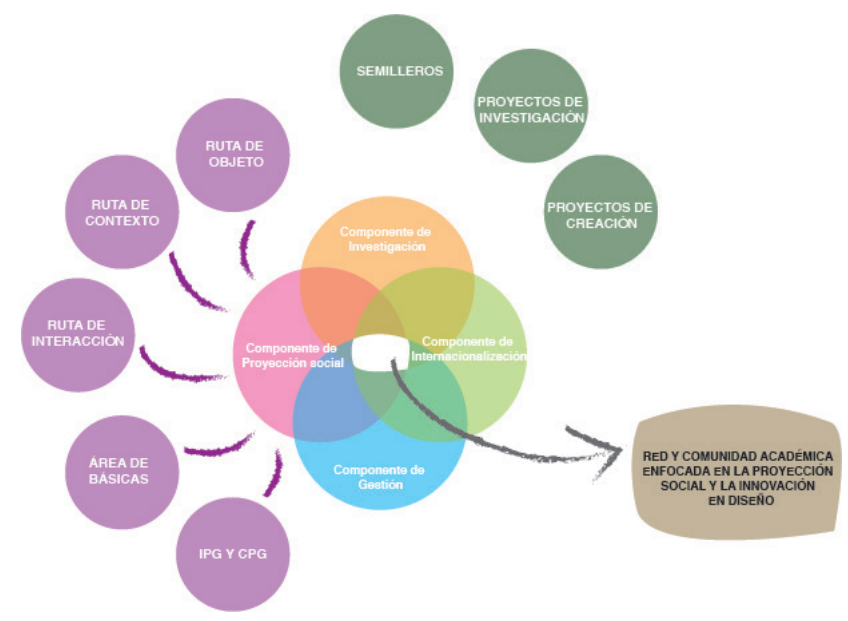

Fuente: Elaboración propia de Zárate (2013).

Imagen 2: Estructura relacional de los componentes y la estructura académica del Programa. Fuente: elaboración propia de Zárate (2013)

Al exponer las relaciones internas y los ejes constitutivos de la red y la comunidad académica es posible mostrar a conti nuación, las relaciones externas que se han generado o que se pretenden generar. En el centro se ubica la red como herramienta 


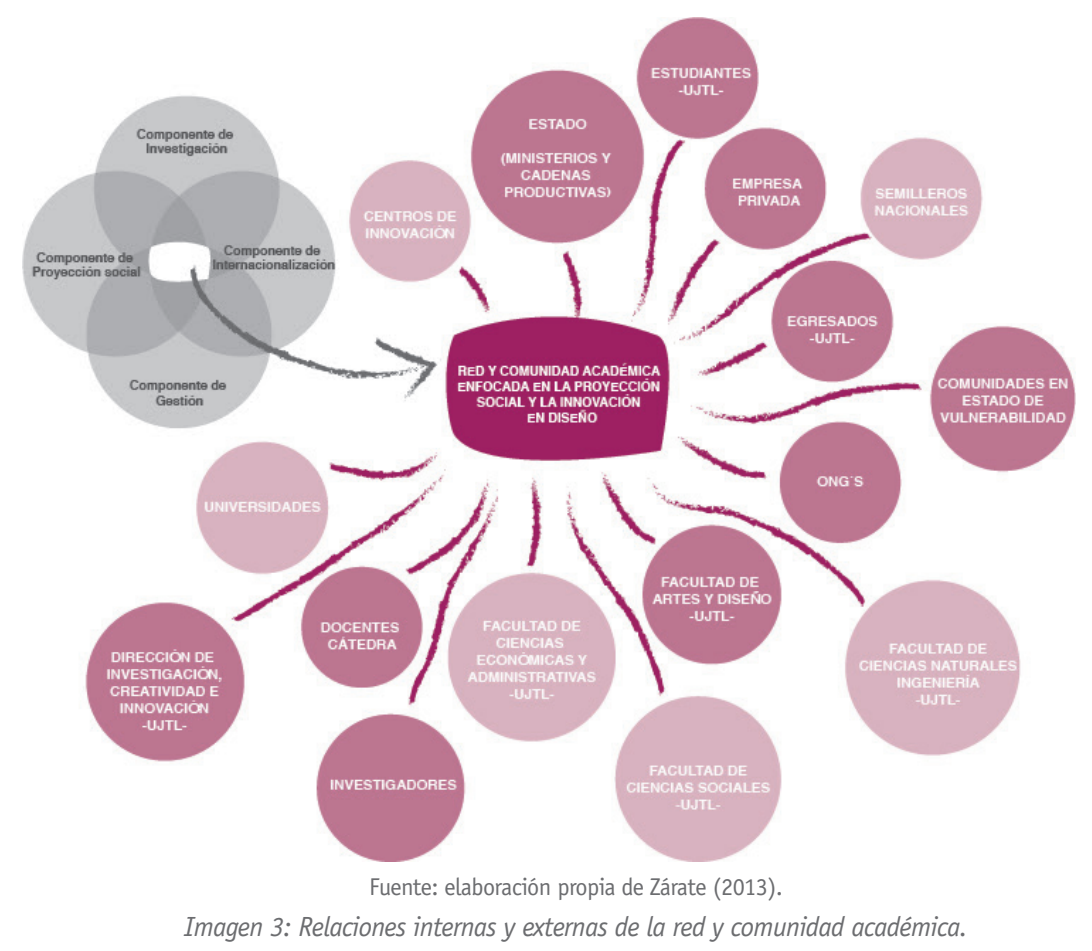

visible articuladora de las relaciones internas y externas. Así, las que están en círculos color más tenues son aquellas relaciones que aun no se han construido pero que son ideales para fortalecer la estructura o que apenas se están construyendo; las que están en círculos de color fuerte son las relaciones que ya están establecidas y con las cuales ya hay proyectos en marcha. El paso que se debe seguir en este punto es la formalización de la cara visible de esta red y la comunidad académica, que se valdrá de diferentes medios, herramientas y tecnologías para cumplir con sus objetivos.

Las partes integradoras para esta red que se proponen utilizar son en su mayoría elementos ya existentes, que pueden enfocarse y aprovecharse para optimizar y agilizar los resultados. En primer lugar, el micrositio del Programa que está albergado en la página de la universidad y al cual acceden desde Internet tanto la comunidad académica tadeísta como la externa. La página de Facebook del Programa, administrada por docentes de tiempo completo y que ha sido de gran utilidad como herramienta de divulgación de información. El boletín de la universidad, que puede apoyar en la divulgación de las actividades mediadas por la red. La página de la universidad para direccionar a los estudiantes desde el portal general a la página de la red $\mathrm{y}$, por último, la videoteca online de la universidad. Todo esto vinculado y direccionado en ambos sentidos con un sitio específico para que la red pueda condensar las actividades exclusivas de la IS en el Programa con sus diferentes involucrados. Este sitio permitirá visibilizar, agilizar, y en algunos casos ejecutar, las actividades enumeradas anteriormente, sobre los temas arriba mencionados; pero además de mostrar lo que se hace dentro y fuera del aula contará con herramientas propias como:

- Encuestas: sobre temas de IS enfocadas al diseño y a la innovación.

- Gestor de ideas: propuestas y postulación de proyectos a tener en cuenta ya sean internos o externos.

- Foros: con docentes, investigadores, estudiantes y gestores participantes en la red.

- Wikis: que permitan la construcción colectiva de un lenguaje común.

- Capsulas de discusión: videos cortos (15 min) con temáticas puntuales de los docentes e investigadores expertos sobre las cuales se pueda generar a futuro un conversatorio en línea o un foro virtual.

- Museo de proyectos: muestra itinerante de los proyectos ya realizados.

- Promoción, divulgación o generación de concursos.

- Bibliografía social: serie de links o adjuntos de artículos ya publicados sobre los proyectos en curso o que sirvan de referente para proyectos de diseño enfocado en la proyección social y la innovación.

Todo esto hace parte de la propuesta que está en este momento en proceso de desarrollo para presentar en busca de la aprobación por parte de la dirección del Programa para iniciar su implementación en el menor tiempo posible. Se espera, entonces, que esta herramienta sirva de apoyo y fortaleza para todo el trabajo ya desarrollado por el componente de Proyección social del programa en los dos años anteriores. 
ARTE \& DISEÑO, ISSN: 1692-8555, Vol. 11 N² 2, Julio - Diciembre 2013 Políticas de innovación social y diseño: entretejido que construye la proyección social de la academia, págs. 5-13

\section{REFERENCIAS BIBLIOGRÁFICAS}

Departamento Nacional de Planeación (DNP), Departamento Administrativo de Ciencia, Tecnología e Innovación (Colciencias), Agencia Nacional para la superación de la pobreza extrema (ANSPE) (2013). Bases conceptuales de una política de innovación social. Bogotá D.C. (Colombia). Recuperado del sitio de internet: http://www.politicadeinnovacionsocial.co/

Programa de Diseño Industrial (2013). PEPA: Proyecto Educativo del Programa Académico. Universidad Jorge Tadeo Lozano, Bogotá D.C. (Colombia).

Universidad Jorge Tadeo Lozano (2011). PEI: Proyecto Educativo Institucional. Universidad Jorge Tadeo Lozano, Bogotá D.C. (Colombia).

\section{FORMA DE CITAR ESTE ARTÍCULO}

Zárate Hernández, J., España Espinoza, J.M. \& Ballesteros Oyaga, S. (2013). Políticas de innovación social y diseño: entretejido que construye la proyección social de la academia. Revista Arte y Diseño Facultad de Arquitectura, Arte y Diseño, Universidad Autónoma del Caribe, Barranquilla. ISSN 1692- 8555 Vol. $11\left(\mathrm{~N}^{\circ} .2\right)$. P.P. 5 - 13 